\title{
GZD824 Inhibits GCN2 and Sensitizes Cancer Cells to Amino Acid Starvation Stress $\$$
}

\author{
Yu Kato, Kazuhiro Kunimasa, Mizuki Takahashi, Ayaka Harada, Ikuko Nagasawa, \\ Masanori Osawa, Yoshikazu Sugimoto, and Akihiro Tomida \\ Cancer Chemotherapy Center, Japanese Foundation for Cancer Research, Koto-ku, Tokyo, Japan (Y.K., K.K., M.T., I.N., A.T.); \\ and Divisions of Chemotherapy (Y.K., M.T., Y.S.) and Physics for Life Functions (A.H., M.O.), Faculty of Pharmacy, Keio \\ University, Minato-ku, Tokyo, Japan
}

Received May 14, 2020; accepted September 24, 2020

\section{ABSTRACT}

Eukaryotic initiation factor $2 \alpha$ (elF2 $\alpha$ ) kinase general control nonderepressible 2 (GCN2) drives cellular adaptation to amino acid limitation by activating the integrated stress response that induces activating transcription factor 4 (ATF4). Here, we found that a multikinase inhibitor, GZD824, which we identified using a cell-based assay with ATF4 immunostaining, inhibited the GCN2 pathway in cancer cells. Indeed, GZD824 suppressed GCN2 activation, elF2 $\alpha$ phosphorylation, and ATF4 induction during amino acid starvation stress. However, at lower nonsuppressive concentrations, GZD824 paradoxically stimulated elF2 $\alpha$ phosphorylation and ATF4 expression in a GCN2-dependent manner under unstressed conditions. Such dual properties conceivably arose from a direct effect on GCN2, as also observed in a cell-free GCN2 kinase assay and shared by a selective GCN2 inhibitor. Consistent with the GCN2 pathway inhibition, GZD824 sensitized certain cancer cells to amino acid starvation stress similarly to ATF4 knockdown. These results establish GZD824 as a multikinase GCN2 inhibitor and may enhance its utility as a drug under development.

\section{SIGNIFICANCE STATEMENT}

GZD824, as a direct general control nonderepressible 2 (GCN2) inhibitor, suppresses activation of the integrated stress response during amino acid limitation, whereas it paradoxically stimulates this stress-signaling pathway at lower nonsuppressive concentrations. The pharmacological activity we identify herein will provide the basis for the use of GZD824 to elucidate the regulatory mechanisms of GCN2 and to evaluate the potential of the GCN2-activating transcription factor 4 pathway as a target for cancer therapy.

\section{Introduction}

The integrated stress response (ISR) plays an important role in cellular adaptation to various stress conditions (Harding et al., 2003, 2000; Ye et al., 2010; Dang, 2012). Under distinct stress conditions, ISR is activated by four eukaryotic initiation factor $2 \alpha$ (eIF2 $\alpha$ ) kinases: general control nonderepressible 2 (GCN2), protein kinase-like endoplasmic reticulum kinase (PERK), double-stranded RNA-dependent kinase, and heme-regulated inhibitor (Quirós et al., 2016). These eIF $2 \alpha$ kinases commonly phosphorylate eIF $2 \alpha$ at S51, thereby reducing general protein synthesis. However, specific mRNAs with an upstream open reading frame, such as activating transcription factor 4 (ATF4), are selectively translated by delaying translation reinitiation via eIF $2 \alpha$ phosphorylation (Lu et al., 2004; Vattem and Wek, 2004). ATF4, a key

This work was supported in part by the Japan Society for the Promotion of Science (JSPS) KAKENHI [Grants 16H04717, 18K19486, and 19H03526] and the Nippon Foundation.

The authors declare no conflicts of interest.

https://doi.org/10.1124/molpharm.120.000070.

S This article has supplemental material available at molpharm. aspetjournals.org. transcription factor for stress adaptation, subsequently drives transcription of genes involved in processes, such as protein folding, amino acid metabolism, and autophagy (Zhu and Thompson, 2019).

Among the eIF2 $\alpha$ kinases, GCN2 is activated in response to amino acid starvation and plays a central role in cellular adaptation to tumor microenvironments (Qiu et al., 1998). Within tumors, cancer cells often undergo amino acid deprivation, partly because abnormal proliferation increases the need for amino acids to produce proteins, lipids, and nucleic acids, and partly because insufficient and disorganized formation of blood vessels leads to a supply shortage of amino acids. Thus, GCN2 can be important for cancer cell survival and tumor development, and consistently, knockout of GCN2 or ATF4 has been shown to decrease tumor growth in vivo (Ye et al., 2010). In addition, the GCN2 arm of the ISR has been recently shown to protect cancer cells from intrinsic stress induced by the $c-M y c$ oncogene by inducing adaptive gene expression and limiting Myc overexpression (Schmidt et al., 2019; Tameire et al., 2019). GCN2 can also be involved in resistance to cancer chemotherapy because sensitization to antitumor agent L-asparaginase is elicited by GCN2 inhibition

ABBREVIATIONS: ATF4, activating transcription factor 4; BCR-ABL, break point cluster region-abelson; BSA, bovine serum albumin; elF2 $\alpha$, eukaryotic initiation factor $2 \alpha$; GCN2, general control nonderepressible 2; GSK, glycogen synthase kinase; ISR, integrated stress response; PERK, protein kinase-like endoplasmic reticulum kinase; siRNA, small interfering RNA. 
in cancer cells that express asparagine synthetase at low levels (Nakamura et al., 2018).

It has been suggested that the GCN2-mediated ISR pathway provides promising targets for cancer therapy, but there are few GCN2 inhibitors available. In this study using a kinase inhibitor library, we screened compounds that inhibit the GCN2 pathway. As a result, we successfully identified GZD824, which is a known BCR-ABL inhibitor (Ren et al., 2013) that prevents GCN2 pathway activation during amino acid starvation stress. We further found that GZD824 directly inhibited GCN2 kinase activity and sensitized certain cancer cell lines to amino acid starvation stress. Our findings from this exploratory study indicate that GZD824 is useful to investigate the role of GCN2 in cellular stress adaptation as a target for cancer therapy.

\section{Materials and Methods}

Cell Culture and Regents. Human fibrosarcoma cell line HT1080 and non-small cell lung cancer cell lines NCI-H460 and A549 were obtained from the American Type Culture Collection (Manassas, VA). HT1080, NCI-H460, and A549 cells were maintained in RPMI-1640 (Wako Pure Chemical Industry, Osaka, Japan) with $10 \%$ fetal bovine serum (Merck, Burlington, MA) as described previously (Saito et al., 2009). All the cells were checked to be mycoplasma-free. GZD824 (Selleck Chemicals, Houston, TX), GCN2iB (MedChemExpress, Monmouth Junction, NJ), GSK2656157 (Merck), halofuginone (Merck), tunicamycin (Nacalai Tesque, Kyoto, Japan), and thapsigargin (Wako) were dissolved in DMSO as stock solutions (final concentration was less than $0.5 \%$ solvent). Histidinol (Merck) was dissolved in sterilized distilled water.

RNA Interference. Silencing of human GCN2, PERK, and ATF4 expression was performed using ON-TARGETplus SMARTpool siRNAs (GCN2: L-005314, PERK: L-004883-00, ATF4: L-005125; GE Healthcare, Little Chalfont, UK) or Silencer Select siRNA (ATF4: s1702; Thermo Fisher Scientific, Waltham, MA) with LipofectAMINE RNAiMAX transfection reagent (Thermo Fisher Scientific). ONTARGETplus SMARTpool and Silencer Select siRNAs were used at 20 and $5 \mathrm{nM}$, respectively. Cells were transfected with each siRNA according to the manufacturers' reverse-transfection protocol.

Immunofluorescence Staining. Cells were seeded in a 96 -well black plate. The cells were treated with reagents at the indicated concentration and time. The cells were fixed in $4 \%$ paraformaldehyde for 15 minutes and then permeabilized with $0.3 \%$ Triton X-100 in $4 \%$ BSA-PBS for 1 hour. The cells were incubated with an anti-ATF4 antibody (11815) (Cell Signaling Technology, Beverly, MA) in 1\% BSAPBS overnight at $4^{\circ} \mathrm{C}$. Then the primary antibody was reacted with an anti-rabbit IgG secondary antibody conjugated with Alexa Fluor-488 (Thermo Fisher Scientific) in 1\% BSA-PBS for 1 hour. The nucleus was counterstained with Hoechst 33342 (Thermo Fisher Scientific). Fluorescence images were obtained by an IN CELL ANALYZER 6000 (GE Healthcare). Quantification of the nuclear ATF4 intensity was performed using IN CELL Developer Toolbox software (GE Healthcare).

Immunoblot Analysis. Immunoblot analysis was performed as described previously (Saito et al., 2009). Briefly, cell lysates were prepared using SDS lysis buffer (62.5 mM Tris-HCl, pH 6.8, 2\% SDS, $50 \mathrm{mM}$ dithiothreitol, and $10 \%$ glycerol). Protein concentrations were determined using a Bio-Rad protein assay (Bio-Rad, Hercules, CA). Protein samples were subjected to SDS-PAGE and subsequently transferred onto a nitrocellulose membrane. Membranes were incubated with primary antibodies against phosphorylated (phospho)GCN2 (Thr899) (ab75836), PERK (ab65142), and eIF2S1 (ab5369) (Abcam, Cambridge, UK) as well as GCN2 (3302), phospho-eIF2 $\alpha$ (Ser51) (3398), ATF4 (11815), and Ribosomal Protein S3 (RPS3) (9538) (Cell Signaling Technology). After washing, the membranes were further incubated with appropriate secondary antibodies [Anti-Mouse IgG, HRP-Linked Whole Ab Sheep or Anti-Rabbit IgG, HRP-Linked Whole Ab Donkey (GE Healthcare)]. The specific signals were detected using Western Lighting plus ECL (Perkin Elmer, Waltham, MA).

In Vitro Kinase Assay. Eight microliters of recombinant GCN2 protein (active; final concentration: $30 \mathrm{nM}$; SignalChem, Richmond, Canada) in reaction buffer (20 mM Hepes, pH 7.4, $5 \mathrm{mM} \mathrm{MgCl}_{2}$, and $1 \mathrm{mM}$ dithiothreitol) was preincubated with $0.2 \mu \mathrm{l}$ of GZD824, dasatinib, or GCN2iB for 60 minutes at $30^{\circ} \mathrm{C}$. Then GCN2 was incubated with $12 \mu \mathrm{l}$ of $2 \mathrm{mM}$ ATP (Sigma) and recombinant eIF2 $\alpha$ (final concentration: $160 \mathrm{nM}$; SignalChem) for 3 hours at $30^{\circ} \mathrm{C}$. These samples were mixed with SDS lysis buffer ( $250 \mathrm{mM}$ Tris-HCl, $\mathrm{pH} 6.8$, $8 \%$ SDS, $40 \%$ glycerol, $0.16 \%$ bromophenol blue, and 20\% 2-mercaptoethanol) and boiled at $100^{\circ} \mathrm{C}$ for 15 minutes. The samples were then subjected to immunoblot analysis as described in 2.4. The immunoblots were quantified using ImageJ (http://rsbweb.nih.gov/ij/) under the Gel Analysis Tool.

Structural Comparison and Preparation of a Docking Model of the GCN2-GZD824 Complex. A docking model of the GCN2-GZD824 complex was prepared using coordinates of the GCN2KAV (Protein Data Bank identification: 6N3L) and ABL-AP24534 (Protein Data Bank identification: 3OXZ) complexes. First, subunit A of the GCN2-KAV complex was superposed on subunit A of the ABLAP24534 complex with the secondary-structure matching-Superpose routine of Coot (Emsley and Cowtan, 2004; Krissinel and Henrick, 2004), resulting in the root-mean-square deviation of $1.81 \AA$. We confirmed that KAV and AP24534 in the complex structure were superposed well and then replaced the coordinates of KAV in the GCN2-KAV complex with those of the superposed AP24534. Second, we replaced the imidazo[1,2-b]pyridazin group of AP23534 to the $1 H$-pyrazolo[3,4-b]pyridine group to obtain GZD824. All molecular graphics in this study were prepared using MacPyMOL version 2.0 (Schrödinger, LLC).

Cell Viability Assay. Cells were seeded in a 96 -well plate and treated with the indicated concentrations of agents. After 48 hours, cell viability was measured by the Cell Titer-Glo luminescent cell viability assay (Promega, Madison, WI). Cell viability is shown as the percentage of the control.

Detection of Apoptotic Cells. Cells were seeded in a 96-well plate and treated with the indicated concentrations of agents. After 48 hours, nuclei were stained with $10 \mu \mathrm{g} / \mathrm{ml}$ Hoechst 33342 (Thermo Fisher Scientific) for 10 minutes. Fluorescence images were acquired with the IN CELL ANALYZER 6000. Apoptotic cells were determined by nuclear condensation and fragmentation. The percentage of apoptotic cells was calculated by dividing the number of apoptotic cells by the total number of cells.

Statistical Analysis. Quantitative results are presented as mean \pm S.D. $(n \geq 3)$. A two-tailed Student's $t$ test was used to evaluate the significance of differences between two groups. A value of $P<0.05$ was considered statistically significant.

\section{Results}

Identification of GZD824 as a GCN2-ATF4 Pathway Inhibitor. To evaluate activation of the ISR promptly and quantitatively, we examined expression levels of ATF4 in nuclei by immunofluorescence staining of HT1080 cells (Fig. 1A). Treatments of cells with halofuginone (prolyl transfer RNA synthetase inhibitor) (D'Aniello et al., 2015) and tunicamycin (N-glycosylation inhibitor) (Gahmberg et al., 1980) for 6 hours induced nuclear ATF4, which was prevented by siRNAs against GCN2 and PERK, respectively. Thus, we found that halofuginone activated the GCN2 arm of the ISR, whereas tunicamycin activated the PERK arm. Using this system, we screened a kinase inhibitor library (355 compounds at $2 \mu \mathrm{M}$ each) and identified GZD824 (Supplemental Fig. 1), a known 
A
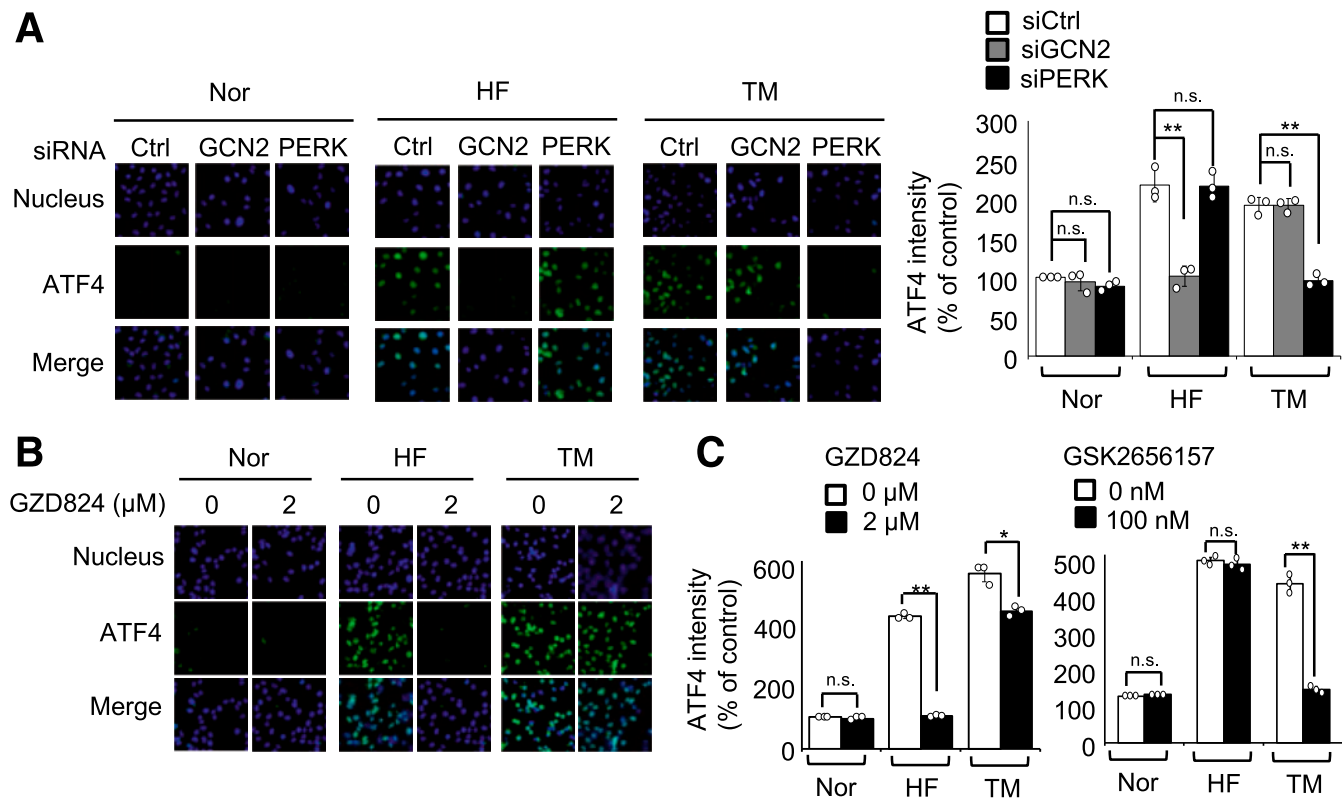

GSK2656157
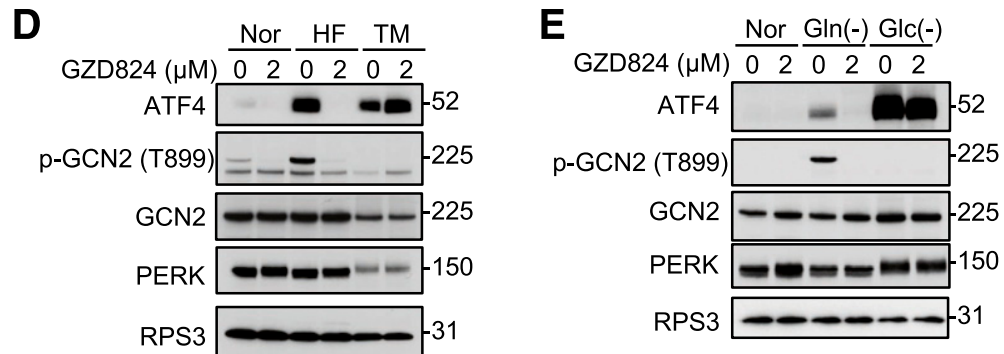

Fig. 1. Identification of GZD824 as a GCN2-ATF4 pathway inhibitor. (A) HT1080 cells were transfected with siRNA against GCN2 and PERK. Then, the cells were treated for 6 hours with halofuginone (HF) and tunicamycin (TM). The cells were fixed and stained with an anti-ATF4 antibody (green), and nuclei were counterstained with Hoechst 33342 (blue) (left). Bars show quantification of the intensity of ATF4 signals in the nucleus. Results are shown as the mean \pm S.D. $(n=3$ ) (right). (B and C) HT1080 cells were treated for 6 hours with $2 \mu$ M GZD824 or 100 nM GSK2656157 under normal (Nor) or stress conditions of HF or TM. The cells were stained with an anti-ATF4 antibody (green) (data not shown for GSK2656157-treated cells), and the intensities of ATF4 signals were quantified as described in (A). (D and E) HT1080 cells were treated for 6 hours with $2 \mu$ M GZD824 under Nor or stress conditions due to $\mathrm{HF}$ or TM addition (D) and for 18 hours with $2 \mu \mathrm{M}$ GZD824 under Nor or stress conditions due to glutamine [Gln (-)] or glucose withdrawal [Glc (-)] (E). Cell lysates were subjected to immunoblot analysis with specific antibodies as indicated. RPS3 was used as the loading control. $* P<0.05 ; * * P<0.001$. Ctrl, control; n.s., not significant; si, siRNA; p-, phosphorylated.

BCR-ABL inhibitor, which preferentially prevents activation of the GCN2 arm compared with the PERK arm.

Indeed, $2 \mu \mathrm{M}$ GZD824 prevented ATF4 induction during halofuginone stress but only marginally during tunicamycin stress in HT1080 cells (Fig. 1, B and C). Conversely, GSK2656157, a specific inhibitor of PERK (Atkins et al., 2013), suppressed ATF4 induction under stress conditions caused by tunicamycin but not halofuginone (Fig. 1C). Immunoblot analysis consistently revealed that GZD824 prevented ATF4 induction under GCN2-activating conditions due to halofuginone addition for 6 hours and glutamine withdrawal for 18 hours but not under PERK-activating conditions due to tunicamycin addition for 6 hours and glucose withdrawal for 18 hours (Fig. 1, D and E). In addition, phosphorylation of GCN2 at T899, a marker of activation, was suppressed by GZD824, but it did not affect the phosphorylation status of PERK, as detected by an upward mobility shift. These results demonstrated that GZD824 inhibited activation of the GCN2-ATF4 pathway in the ISR.

Dual Effects of GZD824 on ATF4 Expression. We next examined the dose-dependent effects of GZD824 on GCN2-ATF4 and PERK-ATF4 pathways. For this purpose, we used histidinol, which mimics amino acid starvation (Zhang et al., 2002), and thapsigargin, a noncompetitive inhibitor of sarco/endoplasmic reticulum $\mathrm{Ca}^{2+}$ ATPase (Urano et al., 2000), as GCN2and PERK-activating stressors, respectively. Treatments of HT1080 cells with these stressors for 4 hours induced ATF4 as shown by immunofluorescence staining (Fig. 2, A and B). In agreement with the above results, the ATF 4 induction by histidinol but not thapsigargin was inhibited by GZD824 in a dose-dependent manner at concentrations ranging from 0.1 to $1 \mu \mathrm{M}$. Furthermore, immunoblot analysis revealed that GZD824 dose-dependently suppressed phosphorylation of GCN2 and induction of ATF4 under histidinol stress (Fig. 2C). These results further showed that GZD824 preferentially inhibited activation of the GCN2-ATF4 pathway of the ISR.

During the above examination, we found that GZD824 by itself induced ATF4 expression under normal conditions, peaking at $0.1 \mu \mathrm{M}$ and attenuating at higher concentrations of 0.3 and $1 \mu \mathrm{M}$ (Fig. 2, A-C; Supplemetal Fig. 2). The ATF4 induction by $0.1 \mu \mathrm{M}$ GZD824 in HT1080 cells occurred with 
A

A Nor
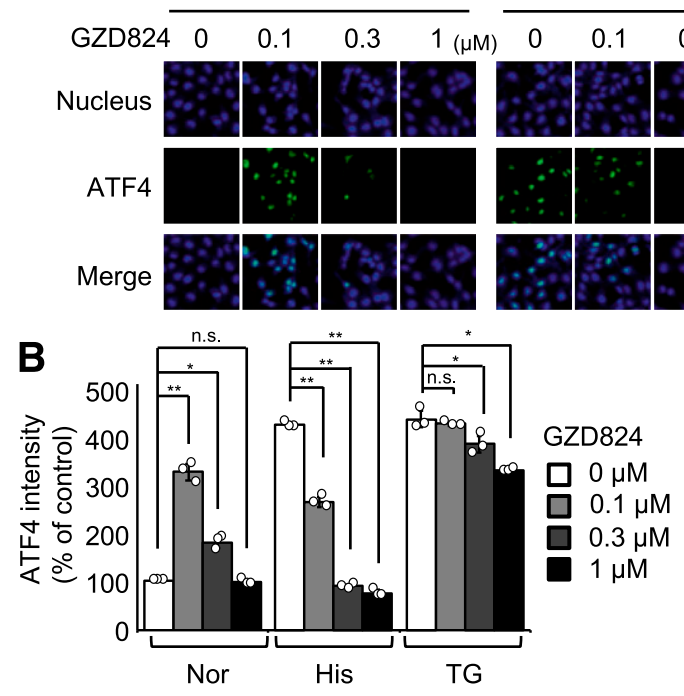

C
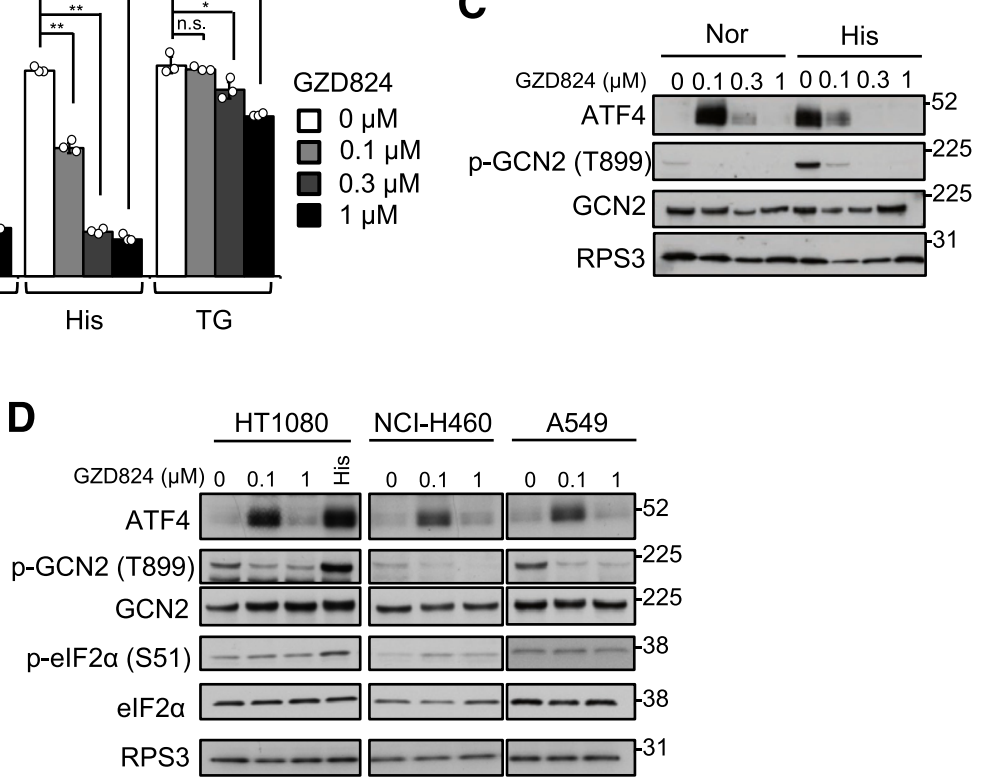

TG

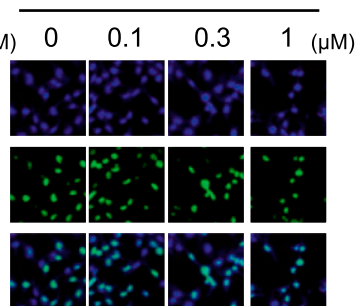

Fig. 2. Dual effects of GZD824 on ATF4 expression. (A and B) HT1080 cells were treated with $0.1,0.3$, or $1 \mu \mathrm{M}$ GZD824 for 4 hours under normal (Nor) or stress conditions of $2 \mathrm{mM}$ histidinol (His) or $300 \mathrm{nM}$ thapsigargin (TG). After 4 hours, the cells were fixed and stained with an anti-ATF4 antibody (green), and nuclei were counterstained with Hoechst 33342 (blue) (A). ATF4 signal intensities were quantified and shown as the mean \pm S.D. $(n=3)$ (B). (C and D) Immunoblot analyses with specific antibodies (as indicated) were carried out using lysates of HT1080 cells treated for 4 hours with $0.1,0.3$, or $1 \mu \mathrm{M}$ GZD824 under Nor or stress conditions of $2 \mathrm{mM}$ His (C) and lysates of HT1080, NCI-H460, and A549 cells that were treated for 4 hours with 0.1 or $1 \mu \mathrm{M}$ GZD824 or $2 \mathrm{mM}$ His (D). RPS3 was used as the loading control. ${ }^{*} P<0.05 ; * * P<0.001$. n.s., not significant; p-, phosphorylated. a slight increase in phosphorylation of eIF2 $\alpha$ S51 but without an increase in phosphorylation of GCN2 T899 (Fig. 2D). In fact, GCN2 phosphorylation was rather decreased by treatment with GZD824. Essentially the same results were obtained using NCI-460 and A549 cells (Fig. 2D). Taken together, GZD824 at low concentrations $(\sim 0.1 \mu \mathrm{M})$ induced ATF4 expression under normal conditions, but at higher concentrations $(0.3-1 \mu \mathrm{M})$, it prevented ATF4 induction under GCN2-activating stress conditions.

GCN2 Kinase as a Direct Molecular Target of GZD824. Although somewhat paradoxically, impeding GCN2 arm activation by GCN2 knockdown impaired ATF4 induction in HT1080 cells treated with $0.1 \mu \mathrm{M}$ GZD824 for 4 hours (Fig. 3A). Thus, a low concentration of GZD824 induced ATF4 likely through a GCN2-dependent mechanism. In line with this finding, such a paradoxical effect of GZD824 on ATF4 expression was recapitulated using GCN2iB, a potent ATP-competitive GCN2 kinase inhibitor that has been reported recently (Nakamura et al., 2018). Indeed, similar to GZD824, GCN2iB elicited ATF4 induction with slightly increased eIF $2 \alpha$ phosphorylation at a low concentration of $0.1 \mu \mathrm{M}$, although it reduced GCN2 phosphorylation dosedependently in the range of $0.1-1 \mu \mathrm{M}$ (Fig. 3B). The GCN2iBelicited ATF4 induction was also impaired by GCN2 knockdown (Fig. 3A). However, unlike GZD824 and GCN2iB, BCR-ABL inhibitors imatinib and dasatinib (Weisberg et al., 2007) had almost no effect on ATF4 expression or GCN2/eIF2 $\alpha$ phosphorylation in HT1080 cells under both normal and stress conditions (Fig. 3C). Thus, in regard to the effects on
GCN2-related signaling pathways, GZD824 shared similar properties with GCN2iB rather than BCR-ABL inhibitors.

We next compared the effects of GZD824, GCN2iB, and dasatinib on GCN2 kinase activity in an in vitro kinase assay consisting of recombinant human GCN2 (192-1024) and eIF2 $\alpha$ (full-length) proteins (Fig. 3D). After incubation with these drugs at graded concentrations in the range of $0.001-1 \mu \mathrm{M}$, GCN2 kinase activity was assessed by phosphorylation levels of eIF2 $\alpha$ S51. In this assay system, GZD824 and GCN2iB similarly inhibited GCN2 kinase activity at 0.1 and $1 \mu \mathrm{M}$, but they rather enhanced the activity at $0.001 \mu \mathrm{M}$. In contrast, dasatinib did not elicit such dose-dependent effects, although it slightly inhibited GCN2 kinase activity. Docking analysis illustrated that GZD824 was accommodated by the inhibitorbinding pocket of the GCN2 kinase (Fig. 3E). These results suggest that, in a way similar to GCN2iB, GZD824 acts on GCN2 directly and elicits biphasic effects on GCN2 kinase activity depending on the concentration.

Cellular Sensitization to GCN2-Activating Stress by GZD824. To examine cellular sensitivity to GZD824, we treated HT1080 cells with this drug $(0.5-1 \mu \mathrm{M})$ for 48 hours in combination with amino acid deprivation-mimicking stressors histidinol and halofuginone. GZD824 alone slightly inhibited cell growth, but it showed a more potent growth inhibitory activity in the presence of either histidinol or halofuginone (Fig. 4A). The combination of GZD824 and histidinol clearly enhanced apoptosis induction as determined by nuclear fragmentation (Fig. 4B). Similarly, GCN2iB prevented ATF4 induction and reduced cell viability during histidinol 
A

HT1080

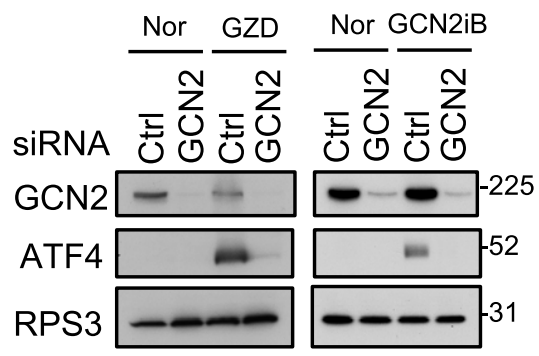

B

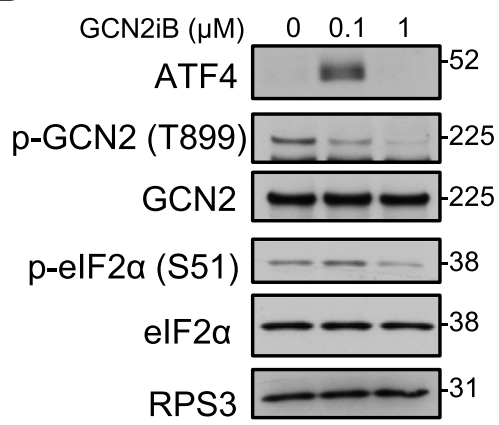

C

HT1080

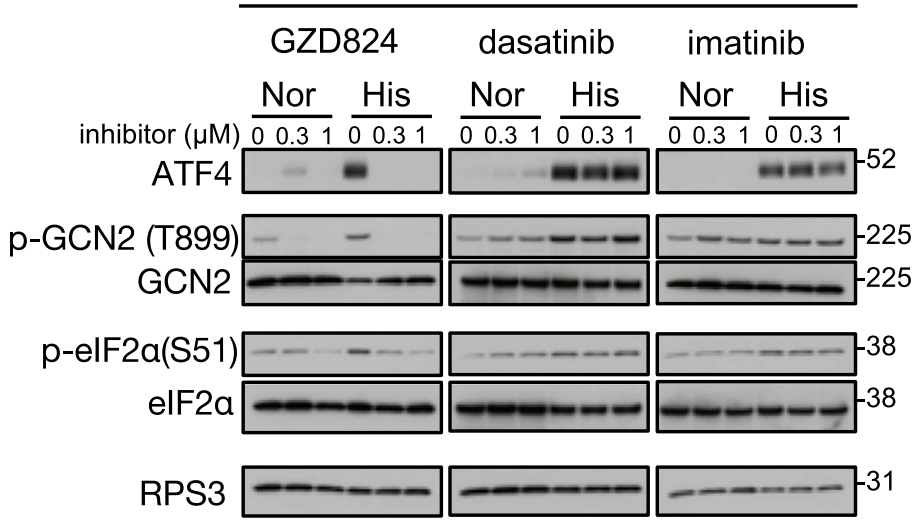

D

\section{GZD824}

GCN2iB

inhibitor 00

GCN2 -+++++

p-elF2a (S51) elF2a

ratio of control (p-elF2 $\alpha /$ elF2 $\alpha)$

E

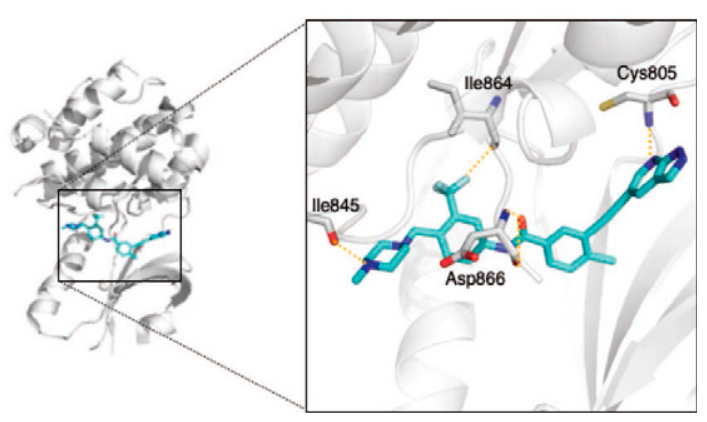

dasatinib
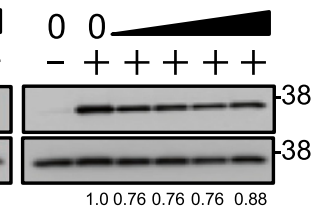

Fig. 3. GCN2 kinase as a direct molecular target of GZD824. Immunoblot analyses with specific antibodies were carried out using lysates prepared after cell or purified protein treatments as follows. RPS3 was used as the loading control. (A) HT1080 cells were transfected with siRNA against GCN2 and then treated for 4 hours with GZD824 (GZD) (100 nM) (left) or GCN2iB (100 nM) (right). (B) HT1080 cells were treated for 4 hours with 0.1 or $1 \mu \mathrm{M} \mathrm{GCN2iB}$. (C) HT1080 cells were treated for 4 hours with GZD824, imatinib, or dasatinib at the indicated concentrations under normal (Nor) or stress conditions of $2 \mathrm{mM}$ histidinol (His). (D) Recombinant GCN2 protein was preincubated for 60 minutes with GZD824, GCN2iB, or dasatinib at $0.001,0.01,0.1$, and $1 \mu \mathrm{M}$. Then the GCN2 proteins were incubated for 3 hours with $2 \mathrm{mM}$ ATP and eIF2 $\alpha$ recombinant protein. (E) Model structure of the GCN2-GZD824 complex. Left: overview of the model structure. Right: binding mode of GZD824 and GCN2. Carbon, nitrogen, and fluorine atoms in GZD824 are shown in cyan, blue, and light blue, respectively. GCN2 residues, which appear to be involved in the interaction with GZD824, are shown as sticks. Putative direct interactions are shown by yellow-dotted lines. Ctrl, control; p-, phosphorylated. stress, although GCN2iB itself had almost no cytotoxicity (Supplemental Fig. 3). These results suggested that GZD824 sensitized HT1080 cells to amino acid starvation stress, possibly by inhibiting the GCN2 arm of the ISR similarly to GCN2iB.

To examine the effects of combined treatment with GZD824 and histidinol in other cell lines, we used NCI-H460 and A549 cells. In both cell lines, GZD824 effectively prevented phosphorylation of GCN2/eIF2 $\alpha$ and induction of ATF4 under 4 hours histidinol stress (Fig. 5A). When both cell lines were treated with GZD824 alone for 48 hours, similar growth inhibition was observed (Fig. 5, B and C). However, sensitization by the combination of GZD824 and histidinol was only seen in NCIH460 cells but not A549 cells. The precise reason remains unknown, but the lack of the sensitizing effect of GZD824 might be related to the fact that A549 cells exhibited intrinsically higher sensitivity to histidinol than NCI-460 and HT1080 cells. Taken together, sensitization to amino acid starvation stress by GZD824 was observed in a manner dependent on the cell type.

Cellular Sensitization to GCN2-Activating Stress by ATF4 Knockdown. In HT1080 and NCI-460 cells wherein GZD824 elicited stress vulnerability, we examined the effects of ATF4 induction on cellular sensitivity to histidinol. For 

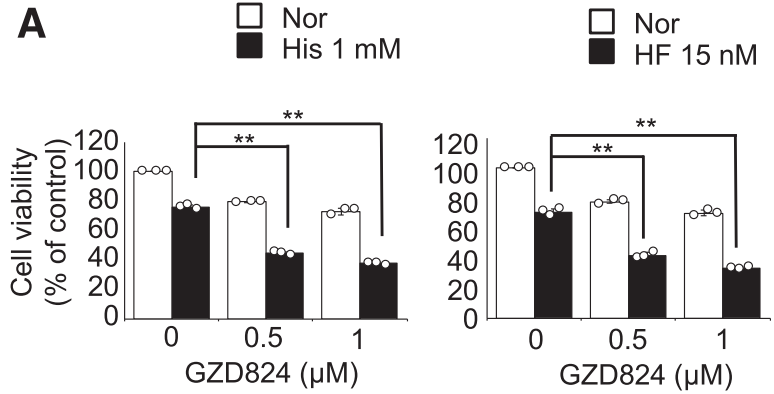

B

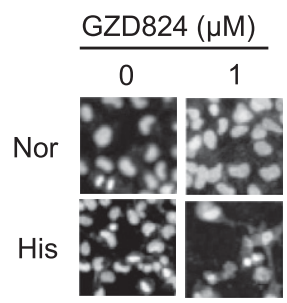

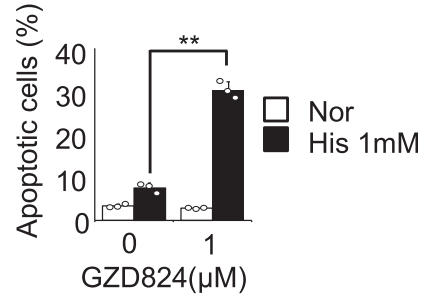

Fig. 4. Sensitization of HT1080 cells to GCN2-activating stress by GZD824. (A) HT1080 cells were treated for 48 hours with GZD824 (GZD) under normal (Nor) or stress conditions of histidinol (His) (left) or halofuginone (HF) (right). Cell viability was determined by the Cell Titer-Glo Luminescent Cell Viability Assay. Results are shown as the mean \pm S.D. $(n=3)$. (B) After treatments of HT1080 cells with GZD824 under Nor or stress conditions of His $(1 \mathrm{mM})$ for 48 hours, cell nuclei were stained with Hoechst 33342 and observed using an IN CELL ANALYZER 6000 . The apoptotic population was calculated and shown by the bar plot. Results are shown as the mean \pm S.D. $(n=3)$. $* * P<0.001$

this purpose, we knocked down ATF4 expression in HT1080 and NCI-460 cells using two different types of siRNAs specific for ATF4 (Fig. 6A; Supplemental Fig. 4A). Although ATF4 knockdown alone for 48 hours had little effect on cell viability except in one experimental set, combined treatment with histidinol led to more potent growth inhibition of HT1080 and NCI-H460 cells (Fig. 6B; Supplemental Fig. 4B). Thus, ATF4 contributed to cell survival during histidinol stress, suggesting that prevention of ATF4 induction plays an important role in GZD824-induced stress vulnerability.

\section{Discussion}

We found that GZD824 effectively prevents activation of the GCN2-ATF4 pathway during amino acid starvation stress. GZD824, which is also known as HQP1351, is an orally available multikinase inhibitor that inhibits imatinibresistant, mutated BCR-ABL and KIT tyrosine kinases. Based on these activities, GZD824 is currently in clinical trials for chronic myelogenous leukemia and in a preclinical development stage for gastrointestinal stromal tumors (Liu et al., 2019). However, it is unlikely that the inhibitory activity against BCR-ABL and KIT tyrosine kinases may be involved in the GCN2 pathway inhibition of the cancer cells in this study. Rather, our in vitro kinase assay revealed that GZD824 inhibits GCN2 kinase activity as potently as GCN2iB, a recently identified, potent, selective GCN2 inhibitor (Nakamura et al., 2018; Fujimoto et al., 2019). Our results also indicated that GZD824 sensitizes certain cancer cells to amino acid starvation stress, possibly by inhibiting GCN2-elicited ATF4 induction. Thus, GZD824 can be considered as a multikinase inhibitor targeting GCN2.

The GCN2-ATF4 pathway promotes cell survival under nutrient-insufficient settings of the tumor microenvironment and plays a major role in tumor growth (Ye et al., 2010). However, the importance of this pathway for cell survival varies depending on the cell and stress types (Ye et al., 2010; Nakamura et al., 2018), as also observed in this study (Figs. 4 and 5). Therefore, to evaluate the potential of the GCN2-ATF4 pathway as a therapeutic target, GCN2 inhibitors would be useful. In fact, identification of the selective GCN2 inhibitor GCN2iB led to the discovery of a synthetic lethal interaction between GCN2 inhibition and asparagine deprivation in a certain type of cancer cell line (Nakamura et al., 2018). Compared with GCN2iB, GZD824 has a different class of chemical structure and inhibits a different, broad spectrum of kinases in addition to GCN2 (Ren et al., 2013). These distinctive features of GZD824 might be useful to discover novel synthetic lethal interactions and widening the potential of the GCN2-ATF4 pathway as a therapeutic target.

It is likely that GZD824 and GCN2iB both paradoxically activate GCN2 at lower concentrations than those that inhibit the GCN2-ATF4 pathway effectively. In fact, low concentrations of GZD824 induced eIF2 $\alpha$ phosphorylation and ATF4 expression in a GCN2-dependent manner in cells under normal nutrient conditions and induced eIF $2 \alpha$ phosphorylation in our in vitro GCN2 kinase assay system (Figs. 2 and 3). These were phenocopied by GCN2iB (Fig. 3), which has been reported to inhibit GCN2 most profoundly among 468 kinases examined (Nakamura et al., 2018). Therefore, these observations suggest that the paradoxical activation of GCN2 stems
A

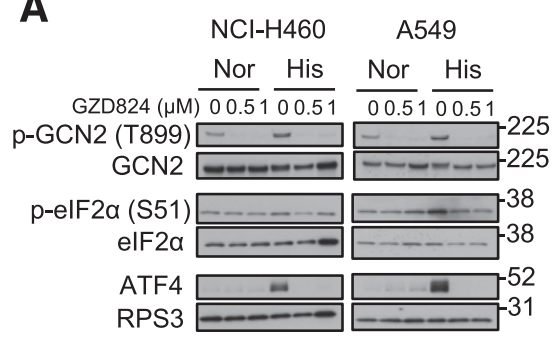

B

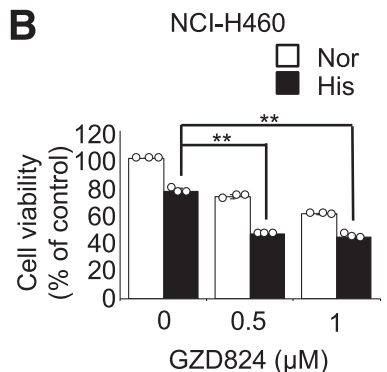

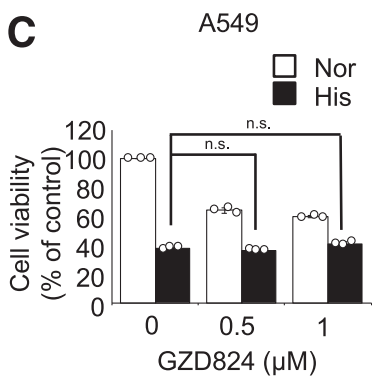

Fig. 5. Sensitization of NCI-H460 cells but not A549 cells to GCN2-activating stress by GZD824. (A-C) NCI-H460 and A549 cells were treated with GZD824 under normal (Nor) or stress conditions of histidinol (His) [1 mM (NCI-H460) or $0.5 \mathrm{mM}$ (A549)] for 4 hours (A) or 48 hours (B and C). After 4 hours of treatment, cell lysates were subjected to immunoblot analysis with specific antibodies as indicated. RPS3 was used as the loading control. (A). After 48 hours of treatment, cell viability was determined by the Cell Titer-Glo Luminescent Cell Viability Assay. Results are shown as the mean \pm S.D. $(n=3)$ (B and C). **P< 0.001. p-, phosphorylated; n.s., not significant. 
A

B

\section{HT1080}

$\square$ Nor

His
$\mathrm{NCl}-\mathrm{H} 460$

Nor
His

Fig. 6. Cellular sensitization to GCN2-activating stress by ATF4 knockdown. (A and B) HT1080 and NCI-H460 cells were transfected with siRNA against ATF4 (Ambion SilencerSelect, $5 \mathrm{nM}$ ) and cultured under normal (Nor) or stress conditions of $1 \mathrm{mM}$ histidinol (His) for 4 hours (A) or 48 hours (B). After 4 hours of treatment, cell lysates were subjected to immunoblot analysis with specific antibodies as indicated. RPS3 was used as the loading control. (A). After 48 hours of treatment, cell viability was determined by the Cell Titer-Glo Luminescent Cell Viability Assay. Results are shown as the mean \pm S.D. $(n=3)($ B). $* * P<0.001$. Ctrl, control; si, siRNA.

from, if not all, the on-target effect of these inhibitors. In this regard, it should be noted that, in contrast to eIF2 $\alpha$ phosphorylation and ATF4 induction, T899 autophosphorylation of GCN2 was decreased conversely by low concentrations of GCN2 inhibitors (Figs. 2 and 3). This observation might support the notion that partial inhibition of GCN2 kinase activity occurs during GCN2-eIF2 $\alpha$-ATF 4 pathway activation in cells treated with low concentrations of GCN2 inhibitors.

So far, GCN2 activation in cell-free systems has been shown to occur by binding of uncharged transfer RNA and, recently, to occur by interactions with ribosomes and the ribosomal P-stalk (Harding et al., 2019). Notably, the latter system indicates that ribosomes stimulate GCN2-dependent eIF2 $\alpha$ phosphorylation with no consistent effect on the phosphorylation status of GCN2 T899 (Harding et al., 2019). Thus, it is likely that T899 autophosphorylation of GCN2 is not always a faithful marker for activation of eIF $2 \alpha$-directed kinase activity of GCN2. However, unlike these cell-free systems, neither uncharged transfer RNA nor ribosomes were added in our in vitro system, suggesting that different mechanisms operated in the drug-induced GCN2 activation. Interestingly, a recent study showed that the human GCN2 kinase domain forms a range of conformational states in the presence of ligands bound to the ATP-binding pocket (Maia de Oliveira et al., 2020). Such an unstable nature may raise the possibility that insufficient or partial kinase inhibition by GCN2 inhibitors leads to an active state of eIF $2 \alpha$-directed kinase activity of GCN2. Future studies, especially structural analysis of GCN2 with various GCN2 inhibitors, together with kinetic analysis of the enzymatic activity will be needed to understand the complicated regulation of GCN2 activity.

In summary, we identified GZD824 as a direct GCN2 inhibitor that prevents activation of the GCN2-ATF4 pathway and sensitizes certain cancer cells to amino acid starvation stress. GZD824 will be useful to elucidate the regulatory mechanisms of GCN2 enzymatic activity and evaluate the potential of the GCN2-ATF4 pathway as a target for cancer therapy. Such information may affect current clinical trials and future clinical development of GZD824.

\section{Acknowledgments}

We thank Mitchell Arico from Edanz Group (https://en-authorservices.edanzgroup.com/) for editing a draft of this manuscript.

\section{Authorship Contributions}

Participated in research design: Kato, Tomida.

Conducted experiments: Kato, Takahashi.

Contributed new reagents or analytic tools: Kunimasa, Nagasawa.
Performed data analysis: Harada, Osawa.

Wrote or contributed to the writing of the manuscript: Kato, Harada, Osawa, Sugimoto, Tomida.

\section{References}

Atkins C, Liu Q, Minthorn E, Zhang SY, Figueroa DJ, Moss K, Stanley TB, Sanders B, Goetz A, Gaul N, et al. (2013) Characterization of a novel PERK kinase inhibitor with antitumor and antiangiogenic activity. Cancer Res 73:1993-2002.

Dang CV (2012) Links between metabolism and cancer. Genes Dev 26:877-890.

D'Aniello C, Fico A, Casalino L, Guardiola O, Di Napoli G, Cermola F, De Cesare D, Tatè R, Cobellis G, Patriarca EJ, et al. (2015) A novel autoregulatory loop between the Gcn2-Atf4 pathway and (L)-Proline [corrected] metabolism controls stem cell identity. Cell Death Differ 22:1094-1105.

Emsley P and Cowtan K (2004) Coot: model-building tools for molecular graphics. Acta Crystallogr D Biol Crystallogr 60:2126-2132.

Fujimoto J, Kurasawa O, Takagi T, Liu X, Banno H, Kojima T, Asano Y, Nakamura A, Nambu T, Hata A, et al. (2019) Identification of novel, potent, and orally available GCN2 inhibitors with type I half binding mode. ACS Med Chem Lett 10: 1498-1503.

Gahmberg CG, Jokinen M, Karhi KK, and Andersson LC (1980) Effect of tunicamycin on the biosynthesis of the major human red cell sialoglycoprotein, glycophorin $\mathrm{A}$, in the leukemia cell line K562 J Biol Chem 255.2169-2175.

Harding HP, Ordonez A, Allen F, Parts L, Inglis AJ, Williams RL, and Ron D (2019) The ribosomal P-stalk couples amino acid starvation to GCN2 activation in mammalian cells. eLife 8:e50149.

Harding HP, Zhang Y, Bertolotti A, Zeng H, and Ron D (2000) Perk is essential for translational regulation and cell survival during the unfolded protein response. Mol Cell 5:897-904.

Harding HP, Zhang Y, Zeng H, Novoa I, Lu PD, Calfon M, Sadri N, Yun C, Popko B, Paules R, et al. (2003) An integrated stress response regulates amino acid metabolism and resistance to oxidative stress. Mol Cell 11:619-633.

Krissinel E and Henrick K (2004) Secondary-structure matching (SSM), a new tool for fast protein structure alignment in three dimensions. Acta Crystallogr D Biol Crystallogr 60:2256-2268.

Liu X, Wang G, Yan X, Qiu H, Min P, Wu M, Tang C, Zhang F, Tang Q, Zhu S, et al. (2019) Preclinical development of HQP1351, a multikinase inhibitor targeting a broad spectrum of mutant KIT kinases, for the treatment of imatinib-resistant gastrointestinal stromal tumors. Cell Biosci 9:88.

Lu PD, Harding HP, and Ron D (2004) Translation reinitiation at alternative open reading frames regulates gene expression in an integrated stress response. $J$ Cell Biol 167:27-33.

Maia de Oliveira T, Korboukh V, Caswell S, Winter Holt JJ, Lamb M, Hird AW, and Overman R (2020) The structure of human GCN2 reveals a parallel, back-toback kinase dimer with a plastic DFG activation loop motif. Biochem $J$ 477: $275-284$.

Nakamura A, Nambu T, Ebara S, Hasegawa Y, Toyoshima K, Tsuchiya Y, Tomita D, Fujimoto J, Kurasawa O, Takahara C, et al. (2018) Inhibition of GCN2 sensitizes ASNS-low cancer cells to asparaginase by disrupting the amino acid response. Proc Natl Acad Sci USA 115:E7776-E7785.

Qiu H, Garcia-Barrio MT, and Hinnebusch AG (1998) Dimerization by translation initiation factor 2 kinase GCN2 is mediated by interactions in the C-terminal ribosome-binding region and the protein kinase domain. Mol Cell Biol 18: 2697-2711.

Quirós PM, Mottis A, and Auwerx J (2016) Mitonuclear communication in homeostasis and stress. Nat Rev Mol Cell Biol 17:213-226.

Ren X, Pan X, Zhang Z, Wang D, Lu X, Li Y, Wen D, Long H, Luo J, Feng Y, et al (2013) Identification of GZD824 as an orally bioavailable inhibitor that targets phosphorylated and nonphosphorylated breakpoint cluster region-Abelson (BcrAbl) kinase and overcomes clinically acquired mutation-induced resistance against imatinib. J Med Chem 56:879-894.

Saito S, Furuno A, Sakurai J, Sakamoto A, Park HR, Shin-Ya K, Tsuruo T, and Tomida A (2009) Chemical genomics identifies the unfolded protein response as a target for selective cancer cell killing during glucose deprivation. Cancer Res 69:4225-4234

Schmidt S, Gay D, Uthe FW, Denk S, Paauwe M, Matthes N, Diefenbacher ME, Bryson S, Warrander FC, Erhard F, et al. (2019) A MYC-GCN2-eIF2 $\alpha$ negative feedback loop limits protein synthesis to prevent MYC-dependent apoptosis in colorectal cancer. Nat Cell Biol 21:1413-1424. 
Tameire F, Verginadis II, Leli NM, Polte C, Conn CS, Ojha R, Salas Salinas C, Chinga F, Monroy AM, Fu W, et al. (2019) ATF4 couples MYC-dependent translational activity to bioenergetic demands during tumour progression. Nat Cell Biol 21:889-899.

Urano F, Wang X, Bertolotti A, Zhang Y, Chung P, Harding HP, and Ron D (2000) Coupling of stress in the ER to activation of JNK protein kinases by transmembrane protein kinase IRE1. Science 287:664-666.

Vattem KM and Wek RC (2004) Reinitiation involving upstream ORFs regulates ATF4 mRNA translation in mammalian cells. Proc Natl Acad Sci USA 101:11269-11274.

Weisberg E, Manley PW, Cowan-Jacob SW, Hochhaus A, and Griffin JD (2007) Second generation inhibitors of BCR-ABL for the treatment of imatinib-resistant chronic myeloid leukaemia. Nat Rev Cancer 7:345-356.

Ye J, Kumanova M, Hart LS, Sloane K, Zhang H, De Panis DN, Bobrovnikova-Marjon E, Diehl JA, Ron D, and Koumenis C (2010) The GCN2-ATF4 pathway is critical for tumour cell survival and proliferation in response to nutrient deprivation. $E M B O \mathrm{~J}$ 29:2082-2096.

Zhang P, McGrath BC, Reinert J, Olsen DS, Lei L, Gill S, Wek SA, Vattem KM, Wek RC, Kimball SR, et al. (2002) The GCN2 eIF2 $\alpha$ kinase is required for adaptation to amino acid deprivation in mice. Mol Cell Biol 22:6681-6688.

Zhu J and Thompson CB (2019) Metabolic regulation of cell growth and proliferation. Nat Rev Mol Cell Biol 20:436-450.

Address correspondence to: Akihiro Tomida, Cancer Chemotherapy Center, Japanese Foundation for Cancer Research, 3-8-31, Ariake, Koto-ku, Tokyo 135-8550, Japan. E-mail: akihiro.tomida@jfcr.or.jp 